\title{
Investigation of horizontal structures at mesospheric altitudes using coherent radar imaging
}

\author{
S. Sommer, G. Stober, C. Schult, M. Zecha, and R. Latteck \\ Leibniz Institute of Atmospheric Physics at the Rostock University, Schloss-Str. 6, 18225 Kühlungsborn, Germany \\ Correspondence to: S. Sommer (sommer@iap-kborn.de)
}

\begin{abstract}
The Middle Atmosphere Alomar Radar System (MAARSY) in Northern Norway $\left(69.30^{\circ} \mathrm{N}, 16.04^{\circ} \mathrm{E}\right)$ was used to perform interferometric observations of Polar Mesosperic Summer Echoes (PMSE) in June 2012. Coherent Radar Imaging (CRI) using Capon's method was applied allowing a high spatial resolution. The algorithm was validated by simulation and trajectories of meteor head echoes. Both data sets show a good correspondence with the algorithm. Using this algorithm, the aspect sensitivity of PMSE was analysed in a case study, making use of the capability of CRI to resolve the pattern within the beam volume. No correction of the beam pattern was made yet. It was found in this case study, that no large variations in the scattering width and the scattering center occured apart from a very short period of time at the upper edge of the PMSE.
\end{abstract}

\section{Introduction}

Polar Mesospheric Summer Echoes (PMSE) are a phenomenon in the middle atmosphere at polar latitudes, where VHF radar waves are backscattered at mesospheric heights (e.g. Balsley et al., 1979; Hoppe et al., 1988). The overall mechanism of the formation of these PMSE is understood and widely accepted. 'Mesospheric neutral air turbulence in combination with a significantly reduced electron diffusivity due to the presence of heavy charged ice aerosol particles (radii $\sim 5-50 \mathrm{~nm}$ ) leads to the creation of structures at spatial scales significantly smaller than the inner scale of the neutral gas turbulent velocity field itself' (Rapp and Lübken, 2004) and therefore scales of the radar's Bragg wavelength. The inner structure of PMSE is still unknown but a field of recent interest (Chilson et al., 2002; Chen et al., 2008). The aspect sensitivity of PMSE is an indicator of the scattering mechanism and describes the backscattered power in dependence on the off-zenith angle of the radar beam. If the power decreases fast with increasing off-zenith angle, the aspect sensitivity is high and it is unlikely an homogenous scattering mechanism and vice versa (Röttger and Vincent, 1978; Hocking et al., 1986). It is essential to understand the aspect sensitivity to use PMSE as a tracer of atmospheric dynamics (Stober et al., 2012). In order to resolve the still unknown inner structures of PMSE, the Middle Atmosphere Alomar Radar System (MAARSY) on the Norwegian Island Andøya started its operation in 2010. MAARSY has a beam width of $3.6^{\circ}$ at $3 \mathrm{~dB}$ and beam steering capabilities with off-zenith angles up to $30^{\circ}$ without generating severe grating lobes. Furthermore the MAARSY antenna can be divided into several sub-arrays to perform interferometric analysis. These capabilities can be used to apply coherent radar imaging (CRI) and to get a deeper insight into the inner structures of PMSE. Coherent Radar Imaging was introduced and established by Kudeki and Sürücü (1991) and Woodman (1997) in atmospheric physics and used by e.g. Yu et al. (2001); Chilson et al. (2002); Chen et al. $(2004,2008)$ to investigate PMSE.

\section{Experimental setup and calculation}

\subsection{Experimental setup}

MAARSY employs an active phased array antenna system located at the island Andøya in Northern Norway $\left(69.30^{\circ} \mathrm{N}\right.$, $\left.16.04^{\circ} \mathrm{E}\right)$. It operates at $53.5 \mathrm{MHz}$ and consists of 433 Yagi antennas, arranged in a circular shape with a diameter of $90 \mathrm{~m}$. It has a half power beam width of $3.6^{\circ}$ and a peak power of about $800 \mathrm{~kW}$. Pulse-to-pulse beam steering is possible to an off-zenith angle up to $30^{\circ}$ (Latteck et al., 2012). For receiving purposes in this experiment, the array was divided in seven sub-arrays (Fig. 1), each consisting of 49 antennas. The receiving channels for the seven sub-arrays have been phasecalibrated as described in Chau et al. (2013). On 21 June 2012, MAARSY was operated in an interferometric mode, 


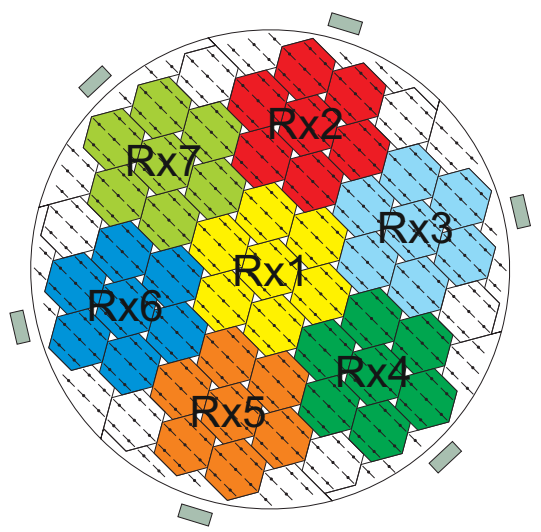

Fig. 1. Sketch of MAARSY. The array is divided into 55 subarrays of hexagonal shape. Seven of contiguous sub-arrays can be combined to a larger structure called an anemone and the seven anemones are colour-coded in the figure above. The boxes around the antenna field are the equipment buildings.

allowing the application of CRI. The sampling range was 75$111 \mathrm{~km}$. Using a pulse length of $1 \mu \mathrm{s}$, the resulting range resolution is $150 \mathrm{~m}$. The pulse repetition frequency was $1250 \mathrm{~Hz}$, a 16 bit complementary coded pulse was transmitted and the received data was stored in 256 points complex time series for each sub-array.

\subsection{Used method}

The data was analysed by using Capon's method of CRI. Capon's method was found to be better than Fourier's method and faster than the Maximum Entropy method (Yu et al., 2000). The imaging method is based on the cross spectral function between the different receivers $i$ and $j$ and denoted by $R_{i j}$. Following Palmer et al. (1998), one can derive the cross correlation matrix in the time domain as

$\mathbf{R}(t)=\left[\begin{array}{cccc}R_{11}(t) & \ldots & R_{1 n}(t) \\ R_{21}(t) & \ldots & R_{2 n}(t) \\ \vdots & & \vdots \\ R_{n 1}(t) & \ldots & R_{n n}(t)\end{array}\right]$

The brightness as an indication for the backscattered power of Capon's method is given by

$\mathbf{B}_{c}(t, k)=\frac{1}{\boldsymbol{e}^{\dagger} \mathbf{R}^{-1} \boldsymbol{e}}$

with

$\boldsymbol{e}=\left[\begin{array}{c}e^{j k \cdot D_{1}} \\ e^{j k \cdot D_{2}} \\ e^{j k \cdot D_{3}} \\ \vdots \\ e^{j k \cdot D_{n}}\end{array}\right]$

where $\boldsymbol{k}$ denotes the wavenumber vector and $\boldsymbol{D}_{\boldsymbol{i}}$ the distance vector of the receiver $i$ with respect to the origin (Palmer et al., 1998), $t$ the time, $\boldsymbol{e}^{\dagger}$ represents the conjugate transpose of $\boldsymbol{e}$ and $\mathbf{R}^{-1}$ the inverse of $\mathbf{R}$.

The algorithm was applied after coherent integration of a time series containing 256 data points. The brightness was mapped on a rectangular grid with a meridional and zonal range of $-4^{\circ}$ to $4^{\circ}$ or $-6^{\circ}$ to $6^{\circ}$ for the simulation, and $-8^{\circ}$ to $8^{\circ}$ for the meteor head echo. The step width is $0.1^{\circ}$. As an initial validation and report of CRI with the MAARSY radar, however, we do not consider the beam weighting effect on the brightness distribution. The beam weighting effect may not be ignored for some circumstances (e.g. Chen et al., 2011; Chen and Furumoto, 2011, 2013).

\section{Verification}

\subsection{Simulation}

Assuming a point target, the backscattered signal can be simulated by a monochromatic wave, which can be written for one sub-array as

$s_{i}(t)=A \cdot e^{j\left(2 \pi f t+\varphi_{i}\right)}+N(t)$

where $A$ is the amplitude, $f$ the transmitted radar frequency, $t$ the time and $\varphi$ the phase. To simulate internal and external disturbances, normally distributed random noise with the standard deviation $\sigma$ and the amplitude $N$ is added for the real and imaginary part independently. This was done for every sub-array for a single target and after that different signal locations were superimposed by adding the different signals of different targets. These signals were used to validate the CRI algorithm. For this simulation, the MAARSY configuration was used, with $f=53.5 \mathrm{MHz}$ and the centers of two adjoining anemones $28 \mathrm{~m}$ apart. The amplitude was set to $A=1$. Furthermore, Gaussian noise with $\sigma=0.1$ was added . The results for three point targets are shown in Fig. 2 with different off-zenith angles. The left figure shows an offzenith angle of $1^{\circ}$. The center of the brightness lies between the scatterers and the single centers can not be separated from each other. The triangular shape is preserved. The right figure shows a simulation with off-zenith angles of $2.5^{\circ}$. All three targets are separated as centers of brightness. Still, some signal is received from the zenith, where no scatterer is located. A more complex distribution of point scatterers is shown in Fig. 3. In contrast to Yu et al. (2000), we assume different centers of scattering. The scatterers are arranged in a sine-shaped way. When we decrease the signal to noise ratio (SNR), the less the shape of the point scatterers is preserved. The SNR was calculated as SNR $=(A / \sqrt{2} \sigma)^{2}$. Hence, the distribution of the brightness depends not only on the distribution of the scatterers but also on the noise level of the observations. The addition of noise for point scatterer in a simulation is necessary, otherwise $\mathrm{R}$ becomes a singular matrix for point scatterers, which cannot be inverted. The ambiguity due to multiple phase cycles becomes a problem for large 

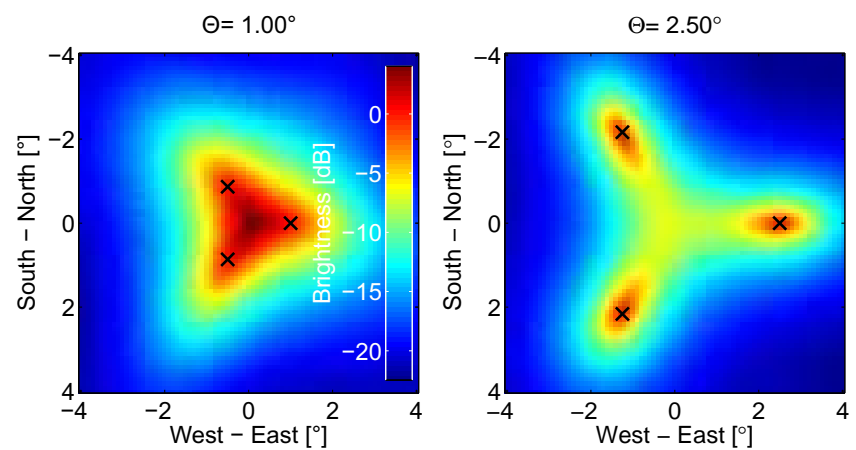

Fig. 2. Simulation of signals with known positions. The three $x$ mark the position of the signal from point targets. Left: Three signals with off-zenith angles of $1^{\circ}$ are superimposed, resulting in one center with the highest brightness in the middle. Right: Three signals with off zenith angles of $2.5^{\circ}$ are superimposed, resulting in three separated centers of brightness.

off-zenith angles. The multiple phase cycles are introduced due to the given baseline length. In Fig. 3, the ambiguity is shown by black lines and determined by the baselines of the used sub-arrays. Even with no noise added, the pattern cannot be reproduced perfectly by the algorithm.

\subsection{Verification using meteor head echoes}

To verify the algorithm not only by simulated but real data, meteor head echoes were used. These echoes result from the plasma around the meteor when it enters the atmosphere. This is a good target due to the small size of the meteors (Westman et al., 2004). An event with two meteors entering the atmosphere with almost perpendicular trajectories were used to validate the CRI algorithm. The trajectories of the meteors are represented in Fig. 4. For this experiment, a pulse length of $49 \mu$ s was used. The range was oversampled with $900 \mathrm{~m}$. For the calculation of the brightness, a set containing $7 \mathrm{~s}$ of data were coherently integrated. The result is shown in Fig. 5 where the brightness distribution for five different heights are shown. The brightness is spread not only horizontally but vertically resulting from the oversampling, but the center of the brightness matches the trajectories. The algorithm is capable of resolving complex structures in real data.

\section{Aspect sensitivity using CRI}

Having validated our CRI algorithm, we performed a PMSE observation in summer 2012 to investigate the aspect sensitivity of this phenomenon for an arbitrarily choosen time. See Sect. 2.1 for the experimental setup. Following Chilson et al. (2002), the brightness distribution in the zenith beam can be regarded as an indicator of the aspect sensitivity during periods when the PMSE are uniform within the beam volume which corresponds to horizontal scales of approximately
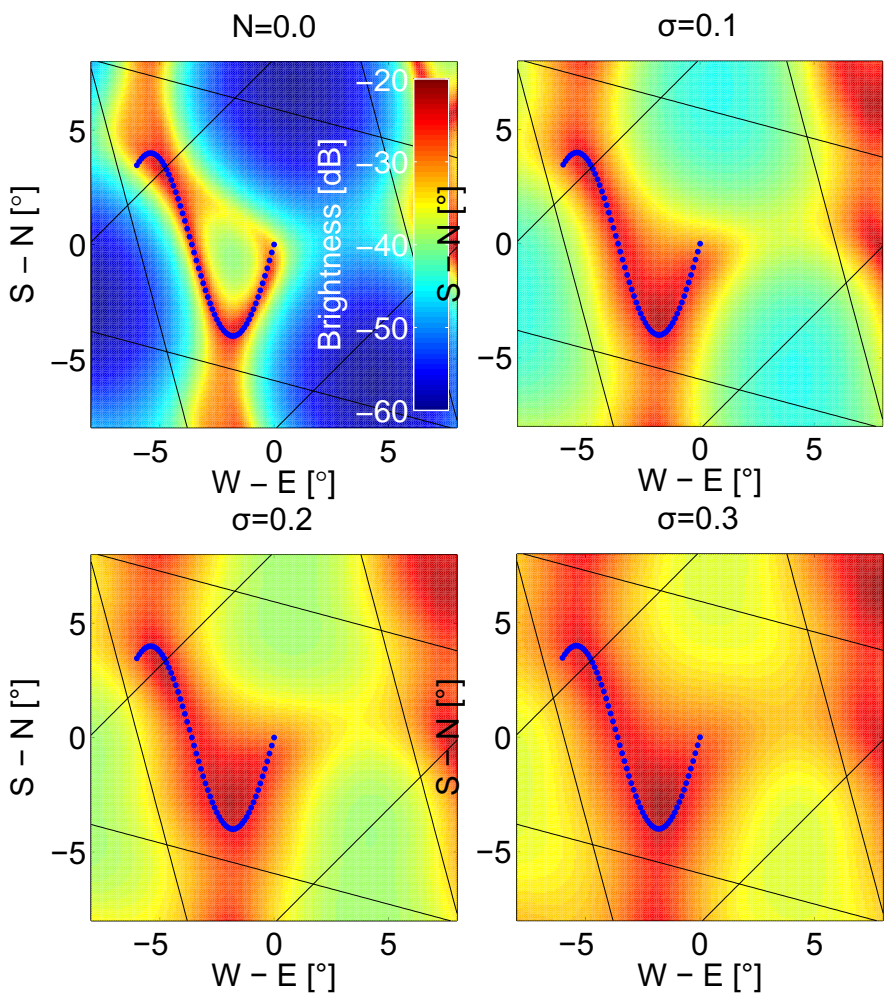

Fig. 3. Simulation of signals with known positions. The dots mark the position of the signal generated by point targets. The black lines indicate the ambiguity limit due to the antenna configuration. Upper left: No noise added reproduce the pattern of the scatterers. Upper right: A SNR of 50 preserves the shape of the scatterer distribution. Lower left: A SNR of 12.5 leads to a broader distribution of brightness. Lower right: A SNR of 5.6 leads to a smeared distribution of brightness.

$5.7 \mathrm{~km}$ at a height of $90 \mathrm{~km}$. Otherwise, the maximum of the brightness must be considered as the mean angle of arrival. The same approach was made for MAARSY. The difference made is that Chilson et al. (2002) applied a two-dimensional Gaussian fit, while we fitted the brightness meridional and zonal with a one-dimensional Gaussian fit of the form

$f(x)=a \cdot \exp \left(-\frac{1}{2}\left(\frac{x-\mu}{\sigma}\right)^{2}\right)$

where $a$ is the amplitude, $\mu$ the deviation from the zenith, in zonal direction denoted by $x$, or meridional direction, denoted by $y$, respectively. $\sigma$ denotes the meridional or zonal width. The CRI algorithm was applied to a time series of nine hours on 21 June 2012 for a height range from $80-89 \mathrm{~km}$ and the brightness was fitted using a Gaussian fit. To avoid the analysis of noise, only data with a maximum brightness $B>75 \mathrm{~dB}$ was included in the analysis. The results for the width and the deviation of the maximum from the zenith of the fit are shown in Fig. 6. 


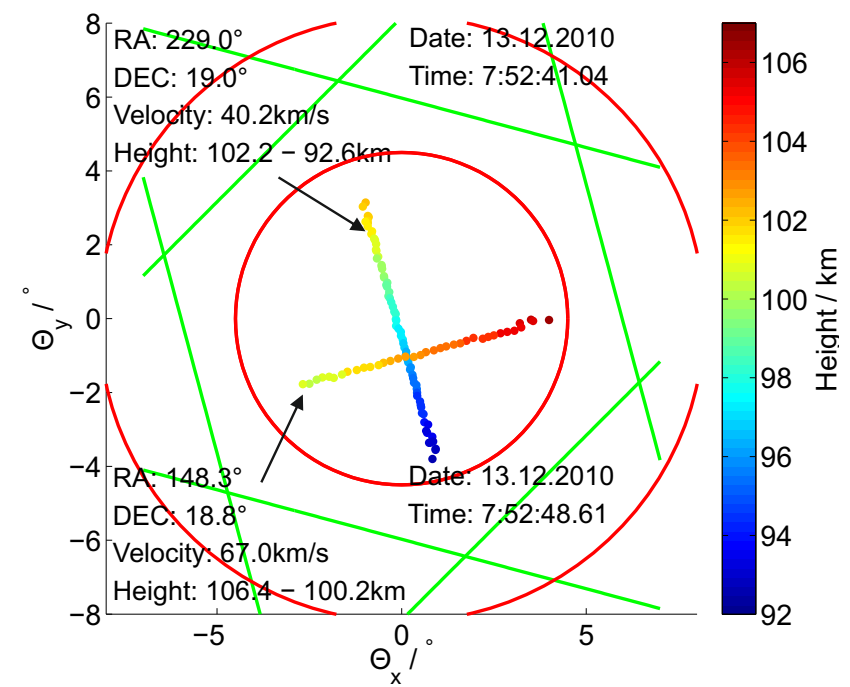

Fig. 4. Two meteors in the zenith beam of MAARSY. These trajectories were calculated by using interferometry. Both meteors enter the atmosphere $7 \mathrm{~s}$ apart and have almost perpendicular trajectories.

The top Fig. 6a shows the range-time power plot of the used time series to give an overview over the PMSE activity during this time. Figures $6 \mathrm{~b}$ and $6 \mathrm{c}$ show the deviation of the fit's maximum in meridional and zonal direction, respectively. Figures $6 \mathrm{~d}$ and $6 \mathrm{e}$ show the width of the fit in meridional and zonal directions. The width of the Gaussian function varies between $\sigma=1^{\circ}$ to $\sigma=3^{\circ}$. This corresponds to a full width at half maximum (FWHM) of FWHM $=2.4^{\circ}$ to FWHM $=7^{\circ}$. Compared with the radar beam width of $\mathrm{FWHM}_{\text {beam }}=3.6^{\circ} \mathrm{we}$ used, the received pattern varies between a broadening of the beam pattern and a smaller received pattern, maybe due to aspect sensitivity. Most returned signals had a width of $\sigma<2.2^{\circ}$. Layers described by Chilson et al. (2002) are not found in this case study, except at about 13:00 UTC, where large deviation over $4^{\circ}$ were found at the upper edge of the PMSE, but no layer shaped broadening in the returned width was found in this time series. It appears that in the lower part of the PMSE the aspect sensitivity is slightly increased compared to the upper part of the PMSE. This is similar to that observed by Chilson et al. (2002).

To inspect the altitudinal variation of aspect sensitivity in more detail, the PMSE was divided into three different parts for each time, where the upper and lower edge are defined by the two lowest and highest ranges of the PMSE and a part between those regions. The variations of the deviations and width for different parts of the PMSE are shown in Fig. 7. The left side shows the deviations of the maximum from the zenith while the right side shows the width of the Gaussian fit. In order to get an overview over the distribution of the scattering center, the expectation values of $\mu_{x}, \mu_{y}, \sigma_{x}$ and $\sigma_{x}$ are shown in Table 1 . The maximum of the brightness is mostly centered around the zenith with no large variations over the different parts of the PMSE. The slight negative de-

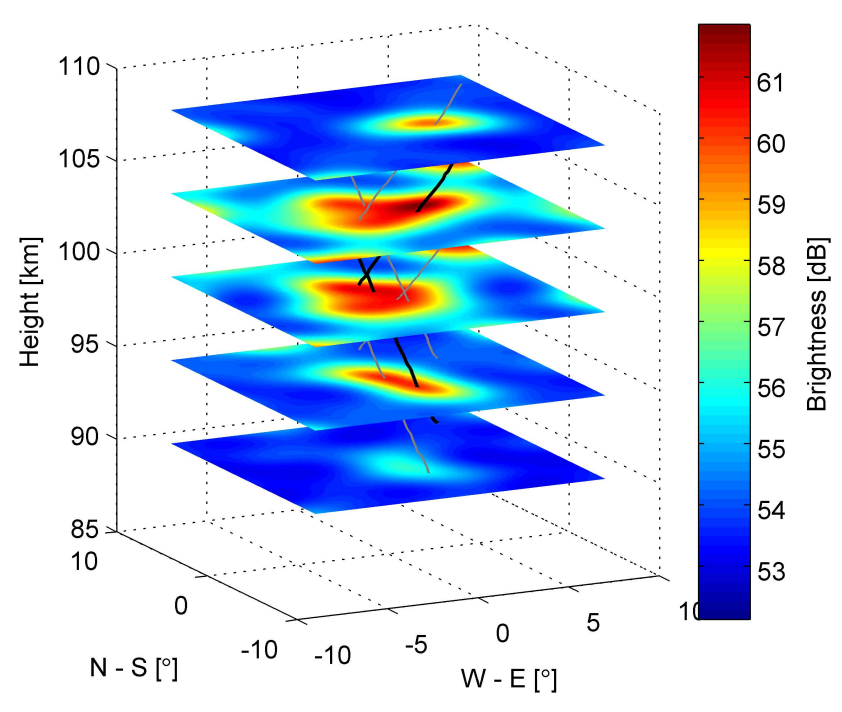

Fig. 5. Meteor trajectories of two meteors. The black lines indicate the center of the meteor trail, the light grey lines indicate the upper and lower limits of the meteor trail due to the used pulse length of $49 \mu$ s. The coloured rectangles represent the brightness in selected height gates. The trajectories and the brightness correspond well.

viation of the scattering centers can result from the PMSE, which might be gently tilted or, although a phase calibration has been made, due to small phase instabilities between the receivers; we need more observations and data to verify this in the future. The width of the Gaussian fit does not vary much over the three different parts of the PMSE, although a slight decrease of aspect sensitivity with height was found in the width of the Gaussian fit in the zonal direction. On the other hand, in the width in meridional direction, no significant change in width was found. The decreasing aspect sensitivity in upper parts of the PMSE was also found by Zecha et al. (2001), who used the Alomar SOUSY VHF radar. Also, Hoppe et al. (1990) measured the aspect sensitivity with the SOUSY VHF radar and they found values of aspect sensitivity between $2^{\circ}$ and $10^{\circ}$ and a tendency of decreasing aspect sensitivity with height.

\section{Summary and conclusions}

A CRI algorithm using Capon's method was applied on PMSE measurements conducted with MAARSY and the algorithm was validated by both synthetic data and meteor head echoes. It was found that the CRI algorithm corresponds well with both the simulation and the trajectories of the meteor head echoes.

After that, the algorithm was used to analyse the aspect sensitivity of PMSE by applying a Gaussian fit to the meridional and zonal slices of the brightness. The brightness distribution can be regarded as an indicator for the aspect sensitivity when we assume, that the PMSE are uniform within the 

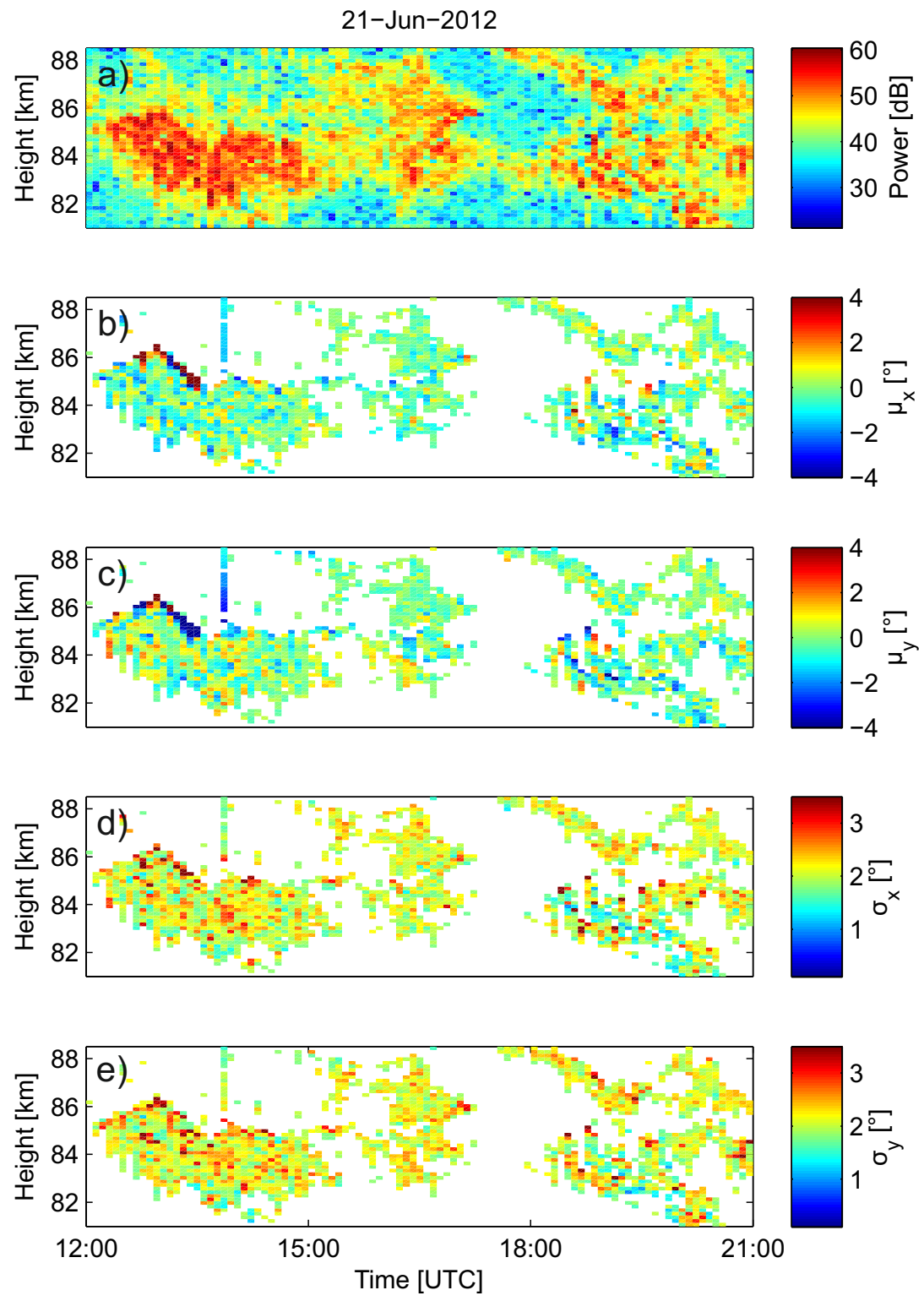

Fig. 6. Range-time pseudocolor plot of: (a) Power plot of the time series. (b) Deviation of the maximal brightness from the zenith in meridional direction. (c) Deviation of the maximal brightness from the zenith in zonal direction. (d) Width of the Gaussian fit in meridional direction. (e) Width of Gaussian fit in zonal direction.

radar beam. Is the PMSE not uniform within the radar beam and the pattern is too small to be resolved by the algorithm, it can only be seen as a mean angle of arrival.

In the dataset used, no layer shaped structures were found, but as a solitary event, in the upper boundary of the PMSE a large deviation from the zenith in both meridional and zonal direction was observed around 13:00 UTC.

Statistical estimates show that the scattering centers differ slightly from the zenith. The slight negative bias could be related to tilted structures in the echo layer, or results from small errors in the phase calibration of the receiving channels. On the other hand, statistical estimates show that the
Table 1. Expectation values of $\mu_{x}, \mu_{y}, \sigma_{x}$ and $\sigma_{x}$. The error is the $\sigma$ standard deviation. The derivation of the scatter centers from the zenith is around zero with a slight negative bias and the width of the brightness distribution is about $2^{\circ}$.

\begin{tabular}{lcccc}
\hline Part of PMSE & $\mu_{x}\left[^{\circ}\right]$ & $\mu_{y}\left[^{\circ}\right]$ & $\sigma_{x}\left[^{\circ}\right]$ & $\sigma_{y}\left[^{\circ}\right]$ \\
\hline Upper edge & $-0.1 \pm 1.3$ & $-0.4 \pm 1.4$ & $2.2 \pm 0.6$ & $2.3 \pm 0.7$ \\
Part between & $-0.1 \pm 1.0$ & $-0.2 \pm 1.0$ & $2.1 \pm 0.5$ & $2.2 \pm 0.5$ \\
Lower edge & $-0.2 \pm 0.8$ & $-0.4 \pm 1.4$ & $2.0 \pm 0.4$ & $2.3 \pm 0.7$ \\
\hline
\end{tabular}



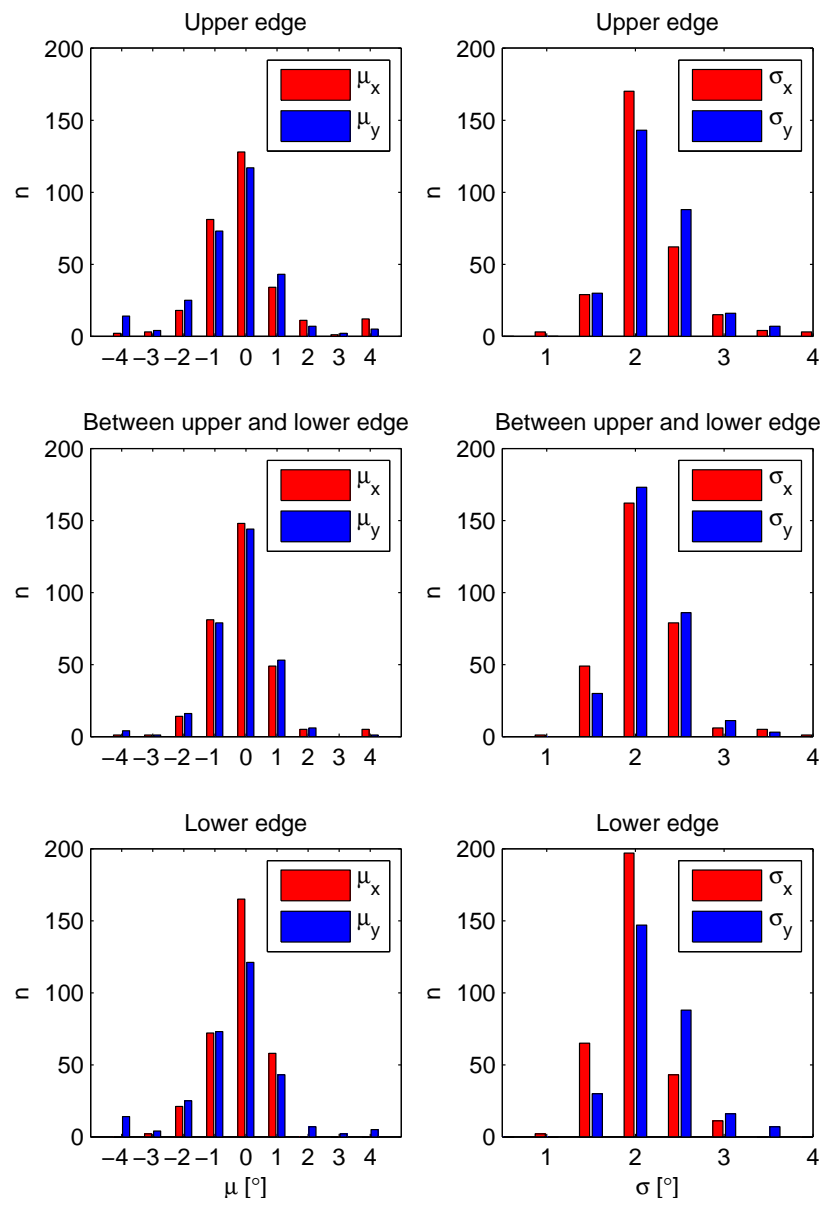

Fig. 7. Bar plots of the deviations from the zenith (left) and the width of the Gaussian fit (right) for different parts of the PMSE. The PMSE was divided in three parts, where the upper edge are the two uppermost heights and the two lowest heights are the lower edge. The part between both regions are pooled as one part. The histograms are fitted with a Gaussian fit (solid line) to get the mean values of $\mu_{x}, \mu_{y}, \sigma_{x}$, and $\sigma_{y}$ in the different parts of the PMSE.

aspect sensitivity, indicated by the width of the brightness distribution, did not vary significantly from the lower to upper parts of the PMSE layer. Only a slight decrease in aspect sensitivity with height was found along the zonal cut.

Further data sets will be analysed in the future to demonstrate the capabilities of CRI with the MAARSY radar, and the beam weighting effects may be taken into account for some circumstances.

Acknowledgements. We like to thank to the Andya Rocket Range for the support while building and operating the MAARSY radar and the IAP personel for keeping the radar running. The radar development was build under grant 01 LP 0802A of Bundesmisterium für Bildung und Forschung. We thank the reviewers for the helpful comments on this paper. Topical Editor Matthias Förster thanks Jenn-Shyong Chen and an anonymous reviewer for their help in evaluating this paper.

\section{References}

Balsley, B., Ecklund, W., Carter, D., and Johnston, P.: Poker Flat MST Radar, 1st Results, Geophys. Res. Lett., 6, 921-924, 1979.

Chau, J. L., Renkwitz, T., Stober, G., and Latteck, R.: MAARSY multiple receiver phase calibration using radio sources, JASTP, doi:10.1016/j.jastp.2013.04.004, 2013.

Chen, J.-S. and Furumoto, J.: A novel approach to mitigation of radar beam weighting effect on coherent radar imaging using VHF atmospheric radar, IEEE Trans. Geosci. Remote Sens., 49, 3059-3070, doi:10.1109/TGRS.2011.2119374, 2011.

Chen, J.-S. and Furumoto, J.: Measurement of atmospheric aspect sensitivity using coherent radar imaging after mitigation of radar beam weighting effect, J. Atmos. Oceanic Technol., 30, 245-259, doi:10.1175/JTECH-D-12-00007.1, 2013.

Chen, J.-S., Hoffmann, P., Zecha, M., and Röttger, J.: On the relationship between aspect sensitivity, wave activity, and multiple scattering centers of mesosphere summer echoes: a case study using coherent radar imaging, Annales Geophysicae, 22, 807817, 2004.

Chen, J.-S., Hoffmann, P., Zecha, M., and Hsieh, C.-H.: Coherent radar imaging of mesosphere summer echoes: Influence of radar beam pattern and tilted structures on atmospheric echo center, Radio Sci., 43, RS1002, 2008.

Chen, J.-S., Chen, C.-H., and Furu, J.: Radar beam- and rangeweighting effects on three-dimensional radar imaging for the atmosphere, Radio Sci., 46, RS6014, 2011.

Chilson, P. B., Yu, T.-Y., Palmer, R. D., and Kirkwood, S.: Aspect sensitivity measurements of polar mesosphere summer echoes using coherent radar imaging, Annales Geophysicae, 20, 213 223, 2002.

Hocking, W., Ruster, R., and Czechowsky, P.: Absolute reflectivities and aspect sensitivities of VHF radiowave scatterers measured with the SOUSY radar, J. Atmos. Terr. Phys., 48, 131-144, doi:10.1016/0021-9169(86)90077-2, 1986.

Hoppe, U., Hall, C., and Röttger, J.: 1st observations of summer polar mesospheric backscatter with a $224 \mathrm{MHZ}$ radar, Geophys. Res. Lett., 15, 28-31, 1988.

Hoppe, U.-P., Fritts, D., Reid, I., Czechowsky, P., Hall, C., and Hansen, T.: Multiple-frequency studies of the high-latitude summer mesosphere : implications for scattering processes, Journal of Atmospheric and Terrestrial Physics, 52, 907-926, doi:10.1016/0021-9169(90)90024-H, http://www.sciencedirect. com/science/article/pii/002191699090024H, 1990.

Kudeki, E. and Sürücü, F.: Radar interferometric imaging of fieldaligned plasma irregularities in the equatorial electrojet, Geophys. Res. Lett, 18, 41-44, doi:10.1029/90GL02603, 1991.

Latteck, R., Singer, W., Rapp, M., Vandepeer, B., Renkwitz, T., Zecha, M., and Stober, G.: MAARSY: The new MST radar on Andoya-System description and first results, Radio Sci., 47, 2012.

Palmer, R., Gopalam, S., Yu, T., and Fukao, S.: Coherent radar imaging using Capon's method, Radio Sci., 33, 1585-1598, 1998.

Rapp, M. and Lübken, F.-J.: Polar mesosphere summer echoes (PMSE): review of observations and current understanding, Atmos. Chem. Phys., 4, 2601-2633, 2004, http://www.atmos-chem-phys.net/4/2601/2004/.

Röttger, J. and Vincent, R.: VHF radar studies of tropospheric velocities and irregularities using spaced antenna techniques, Geo- 
phys. Res. Lett., 5, 917-920, 1978.

Stober, G., Latteck, R., Rapp, M., Singer, W., and Zecha, M.: MAARSY-The new MST radar on Andøya: First results of spaced antenna and Doppler measurements of atmospheric winds in the troposphere and mesosphere using a partial array, ARS, 291-298, doi:10.5194/ars-10-291-2012, 2012.

Westman, A., Wannberg, G., and Pellinen-Wannberg, A.: Meteor head echo altitude distributions and the height cutoff effect studied with the EISCAT HPLA UHF and VHF radars, Ann. Geophys., 22, 1575-1584, 2004,

http://www.ann-geophys.net/22/1575/2004/.
Woodman, R.: Coherent radar imaging: Signal processing and statistical properties, Radio Sci., 32, 2373-2391, 1997.

Yu, T., Palmer, R., and Hysell, D.: A simulation study of coherent radar imaging, Radio Sci., 35, 1129-1141, 2000.

Yu, T.-Y., Palmer, R. D., and Chilson, P.: An investigation of scattering mechanisms and dynamics on PMSE using coherent radar imaging, J. Atmos. Solar-Terres. Phys., 63, 1797-1810, 2001.

Zecha, M., Röttger, J., Singer, W., Hoffmann, P., and Keuer, D.: Scattering properties of PMSE irregularities and refinement of velocity estimates, JASTP, 63, 201-214, 2001. 